\title{
Integrating Innovative Telehealth Solutions into an Interprofessional Team-Delivered Chronic Care Management Pilot Program
}

\author{
Ann M. Taylor, MPH, MCHES; Jennifer Bingham, PharmD; Katherine Schussel, BSN, RN; \\ David Rhys Axon, MPharm, MS; Daniel J. Dickman, MD; Kevin Boesen, PharmD; \\ Rose Martin, PharmD; and Terri L. Warholak, PhD, RPh, FAPhA
}

\begin{abstract}
BACKGROUND: Pharmacist-delivered medication therapy management (MTM) services can improve patient outcomes, yet little is known about outpatient, interprofessional telepharmacy programs.

OBJECTIVE: To evaluate an outpatient, interprofessional telehealth chronic care management (CCM) pilot program.

METHODS: This 6-month program integrated family medicine providers, a university-based medication management telepharmacist, and an interprofessional care coordinator using telehealth solutions for CCM and pharmacy education services. A physician referred patients at risk for medicine-related problems to the telepharmacist. Eligible patients had 3 or more chronic conditions or took at least 5 medications, were aged 18 years and older, and had at least 1 appointment with their primary care provider during the program. The care coordinator met patients in person to facilitate these virtual clinic processes. The telepharmacist conducted a comprehensive medication review (CMR) via video-conferencing technology, providing CCM based on primary diagnosis, current medications and allergies, laboratory results, and previous chart notes. The consultation was documented in the electronic health record (EHR) for provider review and modification in real time.
\end{abstract}

RESULTS: 69 patients received telepharmacy consultations and on-site registered nurse support during the program. Most patients were female (56.5\%), aged $51-70$ years $(60.1 \%)$, Caucasian $(72.4 \%)$, and non-Hispanic/ Latino (71.0\%). Patients had 1-9 chronic conditions, such as hypertension $(82.6 \%)$, diabetes $(56.5 \%)$, hyperlipidemia $(31.9 \%)$, depression $(30.4 \%)$, and osteoporosis $(29.0 \%)$. Most patients $(94.2 \%)$ took at least 5 chronic disease medications, such as statins (11.2\%), nonsteroidal anti-inflammatory drugs $(8.4 \%)$, selective serotonin reuptake inhibitors $(6.5 \%)$, beta blockers $(6.5 \%)$, and calcium channel blockers $(5.6 \%)$. The telepharmacist completed 200 interventions for safety $(49.0 \%)$, vaccines $(24.5 \%)$, care gaps per national consensus guidelines (13.5\%), adherence $(10.0 \%)$, and cost savings (3.0\%). Patients' providers accepted one third $(\mathrm{n}=75,37.5 \%)$ of the telepharmacist recommendations (e.g., monitoring and medication changes).

CONCLUSIONS: This telehealth program constituted an added service for patients while simultaneously filling a gap in on-site pharmacist counseling services. Integrating the telepharmacist and registered nurse was crucial to clinical service provision. The results are encouraging; however, more research must examine the effectiveness of telehealth services in reaching underserved populations, improving patient care, and decreasing health care costs.

J Manag Care Spec Pharm. 2018;24(8):813-18

Copyright $\odot 2018$, Academy of Managed Care Pharmacy. All rights reserved.

\section{What is already known about this subject}

Medication-related problems (MRPs) are responsible for considerable and ever-increasing health care costs.

Innovative approaches to improve health care delivery are needed, such as integrating a telepharmacist as part of an interprofessional team approach in provision of health care services in a virtual setting.

Technologically advanced programs allow pharmacists to reach more patients and reduce MRPs.

\section{What this study adds}

The telepharmacist identified 200 opportunities for improvement of medication therapy quality, safety, or cost (an average of 2.89 per patient), and more than one third of these recommendations were accepted by patients' providers.

The program findings provide initial evidence to support the use of pharmacist-delivered virtual services in order to reach a broader audience, especially patients who would not necessarily receive chronic care management (CCM) services.

A more personalized, telehealth approach may provide the impetus for moving pharmacists closer to health care provider status, allowing them to bill for important patient-care services, including CCM; this could have profound implications for patient care extending far beyond the medication therapy management space.

$\mathrm{M}$ edication-related problems (MRPs) remain an increasing concern in health care today. Estimated morbidityand mortality-related costs for MRPs increased more than 2-fold, from $\$ 76.6$ billion to $\$ 177.4$ billion between 1995 and 2000, respectively. ${ }^{1}$ In 2012, an estimated $\$ 20$ billion were spent on avoidable medication errors, with avoidable pharmacy costs reaching an estimated $\$ 158$ million. $^{2}$ Because these data are older, the costs are likely to be higher today. Providers must now enhance quality, reduce costs, and improve patient outcomes as efficiency and effectiveness move to the forefront. Thus, new, viable solutions must address this escalating problem.

Pharmacists are essential in helping patients by monitoring medications, improving health outcomes, and reducing medication costs ${ }^{3}$ - tasks that echo the Institute for Healthcare 
Improvement's Triple Aim. ${ }^{4}$ Moreover, the pharmacists' important role on the interprofessional team is becoming more well recognized. ${ }^{5}$

Pharmacist-delivered medication therapy management (MTM) services can help patients with chronic conditions achieve better outcomes. ${ }^{6,7}$ MTM encompasses pharmacist services, including comprehensive medication reviews, disease support and coaching, medication safety surveillance, and prevention or wellness services. ${ }^{8}$ MTM services resulting in improved medication utilization and patient education are effective in reducing health care usage and overall related costs. ${ }^{7,9}$

New programs must align with the Affordable Care Act's focus on innovative health care delivery mechanisms and the Center for Medicare and Medicaid Services' push for evaluating novel payment and service models that integrate pharmacists. ${ }^{5}$ Few programs incorporating telepharmacy services are reported in the literature. Yet, as new approaches are explored and evaluated, the respective evidence base will continue to expand. ${ }^{3}$

Innovative models integrating virtual pharmacist services and interprofessional health care teams (e.g., physician and nurse) are a relatively new concept. ${ }^{10-12}$ Previous studies have shown the positive effect of telepharmacy by intervention type ${ }^{13}$; expansion of service hours, available clinical pharmacy services, and cost avoidance ${ }^{14-16}$; and improved outcomes. ${ }^{17}$ To our knowledge, no published studies have evaluated the effect of an interprofessional telepharmacy program in an outpatient clinic setting. Yet, these technologically advanced programs may allow pharmacists to reach populations not routinely served. The objective of this study was to evaluate a virtual, interprofessional chronic care management (CCM) program for patients receiving care at an academic outpatient primary care clinic.

\section{Methods}

\section{Program Design, Patient Sample, and Practice Setting}

This pilot project involved a retrospective record review of patients participating in an interprofessional team-delivered CCM telepharmacy program. Patients were eligible based on having at least 3 chronic conditions or taking 5 or more medications, being aged 18 years or older, and keeping an appointment with their primary care provider at least once during the program period. A total of 69 patients met the criteria and were included in the review.

This program was implemented in a university-based family medicine residency outpatient clinic. The clinic hosted 24 medical residents caring for patients under the direction of 15 board-certified family medicine physicians, most of whom participated in this program. The clinic's services included preventative exams, primary care procedures for common illnesses, prenatal care and delivery, and specialized services (e.g., extended appointments for older adults).
The novel program included an interprofessional health care team of family medicine providers collaborating with a pharmacist and interprofessional care coordinator (e.g, registered nurse) from a university medication management center and utilization of video-conferencing solutions (telepharmacy) in provision of CCM and pharmacy education services.

The attending family medicine physician identified and referred patients at risk for MRPs to the telepharmacist; all referrals were documented in electronic health records (EHR). No formal training was offered, although providers were briefed on the inclusion criteria and virtual appointment process. The clinic was offered 1 morning per week with patient appointments both scheduled in advance or available on a same-day basis.

The telepharmacist and patient were at different locations and yet were able to speak to and see one another via a live broadcast displayed on their respective computer screens. On the appointment day, the interprofessional care coordinator met with patients face to face at the clinic to orient them to the virtual process and answer any questions, facilitate the virtual process with patients and/or their caregivers, and debrief after the session and accompany patients to the waiting/exam room.

For each referral, the telepharmacist conducted a live, virtual comprehensive medication review (CMR), including medication reconciliation, from the patient's medication list, primary diagnosis, allergy profile, laboratory results, previous chart notes, and telepharmacist's direct patient interview. The pharmacist also provided condition-specific patient education and personalized counseling, identified and prevented potential MRPs, and offered other interventions (e.g., improving medication adherence) based on individual need. The telepharmacist documented the consultation directly in the EHR, allowing the provider to view and modify the assessment/ recommendations in real time.

\section{Program Evaluation}

A retrospective EHR review was conducted to evaluate the virtual telepharmacy program. Endpoints of interest included number of interventions per patient, telepharmacist recommendations made, prescriber acceptance of pharmacist recommendations (e.g., gaps in care based on consensus guidelines), safety issue resolution (e.g., drug interactions), and reducing pharmacist-related costs (i.e., travel and clinic space). The university's Institutional Review Board deemed this project a retrospective record review.

\section{Data Collection and Analysis}

The extracted EHR documentation included medicationrelated outcomes (e.g., duplicative medications). All data were de-identified by the telepharmacist including demographic characteristics, past-year health care utilization (i.e., emergency room visits), chronic conditions and medications, recent hospitalizations, and intervention type (e.g., adherence and safety). 
Data analysis was conducted using Microsoft Excel, version 15.26 (Microsoft, Redmond, WA). This pilot project included descriptive statistics only.

\section{Results}

A total of 69 patients met the inclusion criteria and received telepharmacist consultations, with the on-site support from the care coordinator. Patients received these services during the 6-month pilot program.

A diverse group of patients received the telepharmacistdelivered CCM services. More than half were female (56.5\%), and the majority were aged $51-70$ years (60.1\%), with a mean age of 61.7 years. The racial and ethnic mix of patients was more homogenous-most were Caucasian (72.4\%) and of nonHispanic/Latino origin (71.0\%; Table 1).

Patients receiving telepharmacist services were also monitored for chronic conditions, chronic disease medications taken, and health care usage. The number of chronic conditions per patient ranged from 1 to 9 , including hypertension (82.6\%), diabetes (56.5\%), hyperlipidemia (31.9\%), depression (30.4\%), and osteoporosis (29.0\%). The vast majority (94.2\%) were taking at least 5 chronic disease medications for their ailments. Of the top 5 drug classes taken, statins were most common (11.2\%), followed by nonsteroidal anti-inflammatory drugs (8.4\%), selective serotonin reuptake inhibitors (6.5\%), beta blockers (6.5\%), and calcium channel blockers (5.6\%; Table 2).

Regarding health care utilization, $37.7 \%(n=26)$ of patients had used emergency department services 1-3 times in the past year, with a maximum of 10 reported visits in the past year. Past-year physician visits varied considerably (range $=1-29$ ), with a mean (standard deviation [SD]) of $9(S D=6.4)$ visits per patient. Six patients also reported hospitalizations in the 10 days prior to the CMR. Most patients reported having multiple health insurance providers. The most common health insurance provider was Medicaid $(n=48,43.6 \%)$; other providers included Medicare $(n=40,36.4 \%)$, commercial ( $n=17,15.5 \%)$, federal employee or military $(n=4,3.6 \%)$, and joint Medicare and commercial ( $\mathrm{n}=1,0.9 \%)$.

The telepharmacist completed 200 clinical interventions related to safety ( $n=98,49.0 \%)$, vaccines ( $n=49,24.5 \%)$, gaps in care based on national consensus guidelines ( $n=27,13.5 \%)$, adherence $(n=20,10.0 \%)$, and cost savings $(n=6,3.0 \%)$. The majority ( $\mathrm{n}=132,66.0 \%)$ of pharmacist interventions made ranged from 1 to 4 per patient; the most interventions made was 8 for 1 patient. The average number of interventions made was 2.89 per patient. However, 1 patient received no telepharmacist interventions.

Of the 200 telepharmacist recommendations made, more than one third $(n=75,37.5 \%)$ were accepted by providers, with the physician accepting more recommendations than the residents. Provider acceptance of pharmacist recommendations

\begin{tabular}{|c|c|}
\hline \multicolumn{2}{|l|}{ TABLE 1} \\
\hline \\
\hline Female & $39(56.5)$ \\
\hline Male & $30(43.5)$ \\
\hline Age, mean (SD) & $62(12.2)$ \\
\hline \multicolumn{2}{|l|}{ Race, n (\%) } \\
\hline African American & $5 \quad(7.2)$ \\
\hline White or Caucasian & $50(72.4)$ \\
\hline Other/unknown & $14(20.3)$ \\
\hline \multicolumn{2}{|l|}{ Ethnicity, n (\%) } \\
\hline Non-Hispanic/Latino & $49(71.0)$ \\
\hline Hispanic/Latino & $19(27.5)$ \\
\hline Unknown & $1 \quad(1.5)$ \\
\hline \multicolumn{2}{|l|}{ Top 5 chronic conditions, $\mathrm{n}(\%)$} \\
\hline Hypertension & $57(82.6)$ \\
\hline Diabetes mellitus & $39(56.5)$ \\
\hline Hyperlipidemia & $22(31.9)$ \\
\hline Depression & $21 \quad(30.4)$ \\
\hline Osteoporosis & $20(29.0)$ \\
\hline Chronic medications ( $\geq 5)$ taken, $\mathrm{n}(\%)$ & $65(94.2)$ \\
\hline \multicolumn{2}{|l|}{ Top 5 classes of drugs taken $(n=107), n(\%)^{a, b}$} \\
\hline Statin & $12(11.2)$ \\
\hline Nonsteroidal anti-inflammatory drug & $9 \quad(8.4)$ \\
\hline Selective serotonin reuptake inhibitors & $7 \quad(6.5)$ \\
\hline Beta blocker & $7 \quad(6.5)$ \\
\hline Calcium channel blocker & $6 \quad(5.6)$ \\
\hline \multicolumn{2}{|l|}{ Health care use, n (\%) } \\
\hline Emergency department visits in past year, mean (SD) & $1 \quad(1.7)$ \\
\hline Physician visits in past year, mean (SD) & $(6.4)$ \\
\hline Recent hospitalization in past 10 days, $\mathrm{n}(\%)$ & $6 \quad(8.7)$ \\
\hline \multicolumn{2}{|l|}{ Health insurance provider type $(\mathrm{n}=110), \mathrm{n}(\%)^{\mathrm{c}}$} \\
\hline Commercial & $17(15.5)$ \\
\hline Federal employees/military & $4 \quad(3.6)$ \\
\hline Medicaid & $48 \quad(43.6)$ \\
\hline Medicare & $40 \quad(36.4)$ \\
\hline Medicare/commercial & $1 \quad(0.9)$ \\
\hline \multicolumn{2}{|c|}{$\begin{array}{l}\text { aThe total number of instances of medications identified within all } 5 \text { of these drug } \\
\text { classes }(n=107) \text { served as the denominator for calculating the percentage represen- } \\
\text { tation of each. } \\
\text { bThis noncomprehensive list provides examples of some of the conditions these } \\
\text { medications are used to treat. Statins-high cholesterol and lipid levels; NSAID- } \\
\text { inflammatory conditions (e.g., certain types of arthritis); SSRI-depression, anxiety; } \\
\text { beta blocker-hypertension, coronary artery disease, myocardial infarction; } \\
\text { calcium channel blocker-high blood pressure, abnormal heart rhythm, chest pain. } \\
\text { cMost patients had multiple health insurance providers, thus the total number of } \\
\text { provider types exceeds the total number of patients included in the sample. } \\
\text { NSAID = nonsteroidal anti-inflammatory drugs; SD=standard deviation; } \\
\text { SSRI= selective serotonin reuptake inhibitors. }\end{array}$} \\
\hline
\end{tabular}

was documented in the EHR including adjustment to monitoring precautions, discussing adherence, and vaccine administration. Following provider acceptance of the recommendation(s), subsequent telepharmacist action taken included medication change recommendations, increased monitoring, and patient counseling/education. Of the recommendations accepted by providers, over half of the clinical interventions were safety 


\section{TABLE 2 Number of Patients Receiving Telepharmacist Interventions, Telepharmacist Clinical Recommendations Made, and Respective Incidence of Provider Acceptance of Recommendations}

\begin{tabular}{|c|c|c|c|c|}
\hline \multirow{2}{*}{$\frac{\text { Intervention Type }}{\text { Total }}$} & \multirow[t]{2}{*}{$\begin{array}{c}\text { Patients } \\
\text { Receiving } \\
\text { Pharmacist } \\
\text { Intervention } \\
\mathbf{n} \\
164\end{array}$} & \multirow[t]{2}{*}{$\begin{array}{l}\text { Pharmacist-Patient } \\
\text { Interventions and } \\
\text { Subsequent Pharmacist } \\
\text { Recommendations } \\
\text { for Providers } \\
\mathbf{n} \\
200^{\mathrm{a}}\end{array}$} & \multicolumn{2}{|c|}{$\begin{array}{c}\text { Pharmacist } \\
\text { Recommendations } \\
\text { Accepted by } \\
\text { Providers } \\
\mathrm{n}(\%)\end{array}$} \\
\hline & & & 75 & $(37.5)$ \\
\hline Cost saving (e.g., change to generic medication) & 6 & 6 & 0 & $(0.0)$ \\
\hline Gaps in care (e.g., national guidelines) & 27 & 27 & 11 & $(40.7)$ \\
\hline Adherence & 12 & 20 & 14 & $(70.0)$ \\
\hline Vaccine & 31 & 49 & 10 & $(20.4)$ \\
\hline Safety-related recommendations & 88 & 98 & 40 & $(40.8)$ \\
\hline Monitoring concern (e.g., adverse effects, signs/symptoms of DDI, laboratory values) & 33 & 39 & 14 & $(35.9)$ \\
\hline Therapeutic optimization & 21 & 23 & 11 & $(47.8)$ \\
\hline Other & 14 & 14 & 9 & $(64.3)$ \\
\hline Drug-disease interaction & 6 & 7 & 1 & $(14.3)$ \\
\hline Therapeutic duplication & 5 & 6 & 2 & $(33.3)$ \\
\hline Age-related Beer's List contraindication & 5 & 5 & 2 & $(40.0)$ \\
\hline Drug-drug interaction & 4 & 4 & 1 & $(25.0)$ \\
\hline
\end{tabular}

aThe average number of telepharmacist-delivered interventions was 2.89 per patient.

$D D I=$ drug-drug interaction

related $(n=40,53.3 \%)$; the remaining pertained to adherence $(n=14,18.7 \%)$, gaps in care $(n=11,14.7 \%)$, and vaccines $(\mathrm{n}=10,13.3 \%)$. Interestingly, no providers accepted the costsaving interventions (e.g., change from brand name to generic).

Of the safety concerns identified ( $n=98)$, providers accepted the telepharmacist's recommendations in $40.8 \%(n=40)$ of the circumstances. Of the safety issues identified, those related to monitoring were most common $(n=14, n=35.9 \%)$, while the least frequently accepted were age-related Beers List contraindications $(n=2,40.0 \%)$, drug-drug interactions $(n=1,25.0 \%)$, and drug-disease interactions $(n=1,14.3 \%)$. The Beers Criteria Medication List, created by the American Geriatric Society, is an evidence-based reference tool for providers that lists potentially inappropriate medications for use in older adult populations. ${ }^{18}$

\section{Discussion}

The patients in the pilot program were receiving health care at a university-based, family practice outpatient clinic. Most patients were being treated simultaneously for 3 or more chronic conditions and were taking 5 or more medications. However, this is not surprising given that more than half of these patients $(\mathrm{n}=38,55.0 \%)$ were enrolled in Medicare or were Medicare eligible (aged 61 years or older). Additionally, these findings may also suggest that the younger patients (aged 50 years or younger) receiving care at this residency clinic were less healthy.

The number of physician visits was also not surprising given this predominantly Medicare-eligible population. Seniors are the largest consumers of health-care resources and fill close to one third of all prescriptions. ${ }^{19}$ Also, most seniors have at least 1 chronic medical condition, and more than half of these patients take 5 or more medications regularly, ${ }^{19,20}$ typically requiring more health care resources.

The majority of telepharmacist recommendations were related to safety and included drug interactions (drug-drug, drug-disease, and drug-dietary) and therapeutic duplication. Nevertheless, almost one-tenth were recommendations related to adherence, which suggests that patients with multiple chronic conditions face challenges following prescribed medication regimens. Patient adherence aids were provided, such as using pill boxes, placing medicines on or near something used daily/nightly (e.g., near the toothbrush or on the night table), and keeping a calendar of daily medications. It is well recognized that as the number of medications increases, adherence decreases, ${ }^{21}$ and almost 50\% of medications are not taken as prescribed..$^{22}$ Moreover, pharmacists remain a vital resource in helping patients improve their adherence to longterm therapy.22

Thus, telehealth programs such as this may provide additional opportunities for patients to receive interprofessional CCM to improve medication adherence. Proactive involvement by the primary referring physician and interprofessional care coordinator were key to successful recruitment, patient participation, and program implementation. Our findings parallel Schnur et al's (2014) reported need for a coordinator (often the academic researcher) in managing relationships in virtual care teams to ensure that all interests and milestones are met. ${ }^{23}$ 
The number of intervention recommendations accepted by providers in this project $(37.5 \%)$ is within the range previously reported (20\%-60\%) in the literature. ${ }^{24-25}$ However, none of the telepharmacist's cost-related recommendations were accepted. This is somewhat surprising given the current health care environment and focus on improving care while reducing costs. However, studies have shown that incentive programs that reduce costs can result in improved medication adherence. ${ }^{26,27}$ Further work is needed to investigate the types of interventions that improve providers' acceptance of pharmacist recommendations, to ultimately improve patient adherence.

The interprofessional care coordinator was vital to the initial program buy-in and in coordinating with the clinic staff, playing a critical role in helping patients understand medication therapy management, feel comfortable receiving telephonebased services, and become familiar with the virtual setting. In some instances, interdisciplinary-based interventions are more effective than those limited to remote monitoring only. ${ }^{28}$ Although this project did not use lay health workers, the authors are conducting research on using promotoras in conjunction with telepharmacist-delivered services for underserved patients. To that end, we plan to investigate alternative work flow that maximizes use of supportive personnel to decrease costs and protect professional time.

The benefits of telemedicine and telehealth programs are becoming more recognized, as evidenced by recent legislation. The Telemedicine Reimbursement Parity Act, that took effect in Arizona in 2015, requires insurance coverage for patient-care services provided through telemedicine if insurers would typically pay for these services provided in a traditional clinic or hospital setting. Pharmacist-run call centers and videoconferencing are considered part of telemedicine and telehealth thus, passage of this legislation will eventually facilitate reimbursement for pharmacist-delivered telehealth services. ${ }^{29}$

A formal evaluation was not conducted; however, it is reasonable to speculate that the program resulted in cost savings from the clinic's perspective for these reasons: no additional offsite clinic office space was required and the telepharmacist's time was used more efficiently by working seamlessly from a remote location with full access to the clinic's EHR system. These factors facilitated completion of a more-comprehensive medication assessment and prevented duplication of space and effort. Additionally, Roane et al. (2014) found that EHR review combined with a telephonic MTM program provided a more comprehensive assessment of patient therapy. ${ }^{30}$

Several positive implications of integrating telehealth-based CCM services emerged. First, this virtual program filled a gap in services (i.e., no on-site pharmacy) and enabled the telepharmacist to reach patients who may not have received these services otherwise. This project did not address geographic disparities (e.g., rural-residing patients vs. city dwellers) and access to care for those unable to travel to a clinic. However, the authors are evaluating another program employing telehealth technology to address such issues. Second, pharmacists remain a vital resource in helping patients improve adherence to longterm therapy. ${ }^{22}$ Third, the telepharmacist's and interprofessional care coordinator's integral roles reinforce the importance of collaborative health care teams in clinical service provision to assist patients in managing their chronic diseases. ${ }^{29}$ Fourth, this more personalized, telehealth approach may facilitate moving pharmacists closer to health care provider status, allowing them to bill for patient care services (i.e., CCM). ${ }^{29}$ Furthermore, as providers, pharmacists could have profound implications for patient care extending well beyond the MTM space. Future research must assess objective measures of health outcomes, cost savings, and patient satisfaction.

\section{Limitations}

There were some project limitations. First, the available data were part of the routine telepharmacist-delivered CCM at 1 family medicine clinic. Implementation of similar projects is needed to evaluate the effectiveness of telehealth-related chronic care management in other outpatient settings. Second, the relatively small sample for this retrospective evaluation limits its generalizability. Despite this, the pilot data do provide insight into the potential effectiveness of interprofessional, team-delivered telehealth services in outpatient settings.

\section{Conclusions}

This virtual pilot program offered an added service for patients by providing pharmacist-delivered counseling via an enhanced, more-personalized approach to CCM. It also provides support for pharmacists and interprofessional care coordinators as integral partners in CCM service provision. The results are encouraging; yet it is essential to implement similar programs in diverse populations and settings to better examine their ability to improve patient care and decrease health care costs.

\section{Authors}

ANN M. TAYLOR, MPH, MCHES; DAVID RHYS AXON, MPharm, MS; and TERRI L. WARHOLAK, PhD, RPh, FAPhA, University of Arizona College of Pharmacy, Tucson. JENNIFER BINGHAM, PharmD; KATHERINE SCHUSSEL, BSN, RN; and ROSE MARTIN, PharmD; University of Arizona Medication Management Center, Tucson. DANIEL J. DICKMAN, MD, Banner University Medical Center, Tucson, Arizona, and KEVIN BOESEN, PharmD, SinfonîaRx, Tucson, Arizona.

AUTHOR CORRESPONDENCE: Ann M. Taylor, MPH, MCHES, 1295 N. Martin Ave., P.O. Box 210202, Tucson, AZ 85712-0202. Tel.: 520.626.9081; E-mail: taylor@pharmacy.arizona.edu. 


\section{DISCLOSURES}

External funding from SinfonîaRx was used to help conduct this project. Boesen is employed by SinfonîRx. At the time this project was conducted, Martin was employed at the University of Arizona Medication Management Center but is now employed by SinfonîaRx. The other authors have no disclosures to report.

This original research was presented as a poster at the Academy of Managed Care Pharmacy Nexus 2015; October 26-29, 2015; in Orlando, FL.

\section{ACKNOWLEDGMENTS}

The authors acknowledge Bradley Bingham, PharmD, for his contributions to the preparation of this manuscript.

\section{REFERENCES}

1. Ernst FR, Grizzle AJ. Drug-related morbidity and mortality: updating the cost-of-illness model. J Am Pharm Assoc. 2001;41:192-99.

2. IMS Institute for Healthcare Informatics. Avoidable costs in U.S. healthcare: the $\$ 200$ billion opportunity from using medicines more responsibly. June 2013. Available at: http://www.imshealth.com/files/web/ IMSH\%20Institute/Reports/Avoidable_Costs_in\%20_US_Healthcare/IHII_ AvoidableCosts_2013.pdf. Accessed June 6, 2018.

3. Avalere Health. Exploring pharmacists' role in a changing healthcare environment. May 21, 2014. Available at: http://avalere.com/expertise/ life-sciences/insights/exploring-pharmacists-role-in-a-changing-healthcareenvironment. Accessed June 15, 2018.

4. Institute for Healthcare Improvement. The IHI Triple Aim. 2017. Available at: http://www.ihi.org/Engage/Initiatives/TripleAim/Pages/default.aspx. Accessed June 6, 2018

5. Giberson S, Yoder S, Lee MP. Improving patient and health system outcomes through advanced pharmacy practice. A report to the U.S. Surgeon General. Office of the Chief Pharmacist. U.S. Public Health Service. December 2011. Available at: https://www.accp.com/docs/positions/misc/ Improving_Patient_and_Health_System_Outcomes.pdf. Accessed June 15, 2018.

6. Vira T, Colquhoun M, Etchells E. Reconcilable differences: correcting medication errors at hospital admission and discharge. Qual Saf Health Care. 2006;15(2):122-26

7. Iyer R, Coderre P, McKelvey T, et al. An employer-based, pharmacist intervention model for patients with type 2 diabetes. Am J Health Syst Pharm. 2010;67(4):312-16

8. American Pharmacists Association. APhA MTM Central. What is medication therapy management? 2017. Available at: http://pharmacistsprovidecare. $\mathrm{com} / \mathrm{mtm}$. Accessed June 19, 2018.

9. Wittayanukorn S, Westrick SC, Hansen RA, et al. Evaluation of medication therapy management services for patients with cardiovascular disease in a self-insured employer health plan. J Manag Care Pharm. 2013;19(5):385-95 Available at: https://www.jmcp.org/doi/10.18553/jmcp.2013.19.5.385.

10. Payette C, Desrochers J, Lavoie-Tremblay M, Richer MC. Exploring perceptions of healthcare professionals in the implementation of a new professional role of clinical telehealth coordinator within a university integrated healthcare network. Telemed J E Health. 2010;16(5):614-19.

11. Friesner DL, Scott DM, Rathke AM, Peterson CD, Anderson HC. Do remote community telepharmacies have higher medication errors than traditional community pharmacies? Evidence from the North Dakota telepharmacy project. J Am Pharm Assoc. 2011;51(5):580-90.

12. Lam A. Practice innovations: delivering medication therapy management services via videoconference interviews. Consult Pharm. 2011;26(10):764-74.

13. Sankaranarayanan J, Murante LJ, Moffett LM. A retrospective evaluation of remote pharmacist interventions in a telepharmacy service model using a conceptual framework. Telemed J E Health. 2014;20(10):893-901.
14. Stubbings T, Miller C, Humphries TL, Nelson KM, Helling DK. Telepharmacy in a health maintenance organization. Am J Health Syst Pharm. 2005;62(4):406-10.

15. Young GJ, Rickles NM, Chou CH, Raver E. Socioeconomic characteristics of enrollees appear to influence performance scores for Medicare Part D contractors. Health Aff (Millwood). 2014;33(1):140-46.

16. Garrelts JC, Gagnon M, Eisenberg C, Moerer J, Carrithers J. Impact of telepharmacy in a multihospital health system. Am J Health Syst Pharm. 2010;67(17):1456-62.

17. McFarland M, Davis K, Wallace J, et al. Use of home telehealth monitoring with active medication therapy management by clinical pharmacists in veterans with poorly controlled type 2 diabetes mellitus. Pharmacotherapy. 2012;32(5):420-26.

18. Campanelli CM. American Geriatrics Society updated Beers Criteria for potentially inappropriate medication use in older adults: the American Geriatrics Society 2012 Beers Criteria update expert panel. J Am Geriatr Soc. 2012;60(4):616-31.

19. Centers for Disease Control and Prevention, The Merck Institute for Aging and Health. The state of aging and health in America 2004. Available at: https://www.cdc.gov/aging/pdf/State_of_Aging_and_Health_in_ America_2004.pdf. Accessed June 6, 2018.

20. Kaufman DW, Kelly JP, Rosenberg L, Anderson TE, Mitchell AA. Recent patterns of medication use in the ambulatory adult population of the United States: The Slone survey. JAMA. 2002;287(3):337-44.

21. Vlasnik JJ, Aliotta SL, DeLor B. Medication adherence: factors influencing compliance with prescribed medication plans. Case Manager. 2005; 16(2):47-51.

22. Sabaté E, ed. Adherence to long-term therapies: evidence for action. World Health Organization. 2003. Available at: http://apps.who.int/iris/bitstream/10665/42682/1/9241545992.pdf. Accessed June 6, 2018

23. Schnur ES, Adams AJ, Klepser DG, Doucette WR, Scott DM. PCMHs, ACOs, and medication management: lessons learned from early research partnerships. J Manag Care Pharm. 2014;20(2):201-05. Available at: https:// www.jmcp.org/doi/10.18553/jmcp.2014.20.2.201

24. Michaels NM, Jenkins GF, Pruss DL, Heidrick JE, Ferreri SP. Retrospective analysis of community pharmacists' recommendations in the North Carolina Medicaid medication therapy management program. J Am Pharm Assoc. 2010;50(3):347-53.

25. Perera PN, Guy MC, Sweaney AM, Boesen KP. Evaluation of prescriber responses to pharmacist recommendations communicated by fax in a medication therapy management program (MTMP). J Manag Care Pharm. 2011;17(5):345-54. Available at: https://www.jmcp.org/doi/10.18553/ jmcp.2011.17.5.345.

26. Clark B, DuChane J, Hou J, Rubinstein E, McMurray J, Duncan I. Evaluation of increased adherence and cost savings of an employer valuebased benefits program targeting generic antihyperlipidemic and antidiabetic medications. J Manag Care Pharm. 2014;20(2):141-50. Available at: https:// www.jmcp.org/doi/10.18553/jmcp.2014.20.2.141.

27. Chen SY, Shah SN, Lee YC, Boulanger L, Mardekian J, Kuznik A. Moving branded statins to lowest copay tier improves patient adherence. J Manag Care Pharm. 2014;20(1):34-42. Available at: https://www.jmcp.org/ doi/10.18553/jmcp.2014.20.1.34

28. Feltner C, Jones CD, Cene CW, et al. Transitional care interventions to prevent readmissions for persons with heart failure: a systematic review and meta-analysis. Ann Intern Med. 2014;160(11):774-84.

29. Marotta R. The state of provider status: an update for pharmacy students. Pharm Times. February 22, 2016. Available at: http://www.pharmacytimes. com/publications/career/2016/pharmacycareers_february2016/the-state-ofprovider-status-an-update-for-pharmacy-students. Accessed June 19, 2018.

30. Roane TE, Patel V, Hardin H, Knoblich M. Discrepancies identified with the use of prescription claims and diagnostic billing data following a comprehensive medication review. J Manag Care Pharm. 2014;20(2):165-73. Available at: https://www.jmcp.org/doi/10.18553/jmcp.2014.20.2.165. 